\title{
Heartbeats: music recommendation system with fuzzy inference engine
}

\author{
Vinothini Kasinathan ${ }^{1}$, Aida Mustapha ${ }^{2}$, Tan Sau Tong ${ }^{3}$, Mohamad Firdaus Che Abdul Rani ${ }^{4}$, \\ Nor Azlina Abd Rahman \\ ${ }^{1,3,4,5}$ School of Computing, Asia Pacific University of Technology and Innovation, Taman Teknologi Malaysia, Malaysia \\ ${ }^{2}$ Faculty of Computer Science and Information Technology, Universiti Tun Hussein Onn Malaysia, Malaysia
}

\begin{tabular}{l}
\hline Article Info \\
\hline Article history: \\
Received Nov 1, 2018 \\
Revised Feb 6, 2019 \\
Accepted Mar 5, 2019 \\
\hline
\end{tabular}

\section{Keywords:}

Fully logic

Recommendation system

User profiling

\begin{abstract}
In developing a music recommendation system, there are several factors that can contribute to the inefficiency in music selection. One of the problems persists during the music listening is that common music playing application lacks the ability to acquire context of the user. Another problem that common music recommendation system fails to address the emotional impact of the recommended song. To address this gap, this paper presents a music recommendation system based on fuzzy inference engine that considers user activities and emotion as part of the recommendation parameters. The system includes building a smart music recommendation system that has user profiling capabilities to recommend correct songs based on the user's preferences, mood and time. Findings of the this paper have shown that Heartbeats's fuzzy inference engine has successfully achieved its aim, which is to improve users' music listening experience by giving suitable song recommendation based on user context situation.
\end{abstract}

Copyright $(0) 2019$ Institute of Advanced Engineering and Science. All rights reserved.

\section{Corresponding Author:}

Vinothini Kasinathan,

School of Computing,

Asia Pacific University of Technology and Innovation,

Taman Teknologi Malaysia, 57000 Kuala Lumpur, Malaysia.

Email: vinothini@.apu.edu.my

\section{INTRODUCTION}

Music is sound that has been given upon rhythm and energy to invoke feelings on the listener [1]. Humans often find that music is emotionally expressive. There is a variety of reasons that contribute to music listening and the choice of music [2]. Among the common factor includes the improvement in productivity, as stress relief, preferences and emotion. Each aspect should be studied thoroughly to gain insights for building the music recommendation algorithm to cater to users in a psychological manner. Music is also known to have impact on human's ergogenic effect such as among swimmers [3]. The experiment tested the swimmer to swim under two conditions, one with asynchronous music and one with no music control. It was found that no music control condition shows a high time trial but low state of motivation. However, motivational music used in the experiment shows little difference in performance.

Another experiment by [4] showed that tempo of a pop music affects post-treadmill exercise recovery time. The results showed that pop song with low beat per minute has the beset effect in heartbeat recovery. This suggest users should have extra consideration in choosing music for listening when performing physical workouts. Aside from being able to improve the listener's productivity, music is also known to be able to heal the listener's mind. Many people choose to listen to music when they are feeling depressed or under stress because it makes them feel better and easier to cope with. Music treatment during an awake craniotomy is 
proven to be effective in reducing the patient's stress level and anxiety [5]. This concludes that music has psychological effect on human mind, hence important to have a music recommendation application.

Music is also a great tool to recover from mental exhaustion. After listening to relaxing music, humans can easily recover themselves from a mentally daunting task. An experiment is performed by [6] showed that music is responsible for relieving mental exhaustion from a complicated task. There is a demand for relaxation music for people who are working or studying a lot, thus the music recommendation algorithm should consider the possibility. Joyful music such as the pop can improve people mood as well [7]. Recall that [2] measured emotional responses produced by humans while listening to music in the context of arousal and valence. This is important in deciding factor types of music to recommend when users are coping with certain emotions. The most common way to categorize music into a different degree of emotional responses is by their genre. A music genre is a representation of the style or aesthetic pattern of the music's beat, rhythm and instrument and a product of the time of the culture. The similarities between the music of within the same genre will evoke similar emotions to the listener.

In developing a music recommendation system, there are several factors that can contribute to the inefficiency in music selection. One of the problems persists during the music listening is that common music playing application lacks the ability to acquire context of the user. There are many different factors to consider that can improve the quality of the music recommendation. One such example, is the context of the activity the user is doing while listening to music. If the user is working, they might need a music track that does not take their concentration away, which is the antithesis of a good music recommendation. Another problem that common music recommendation system fails to address the emotional impact of the recommended song. One of the top three causes of stress is work [8]. Excess stress can lower one's performance and health [8]. Therefore, many people try to find ways to relieve stress to avoid crumble under pressure. Among the methods people choose for stress relief, music has been a pretty common method. Music and relieve a person's anxiety and empowers them to face even more challenge [9]. However, a genre of the music is important as the different genre of music invoke different feelings to humans $[10,11]$ a wrong track will do worse than silence. Therefore, the recommendation algorithm can be improved to tackle such difficulties.

From another perspective, music tracks also need the publicity so that they can be enjoyed by people. However, certain less-known songs will be overshadowed by the trending songs [12-14]. This does not indicate there will be no people enjoy the less-known songs, but it is harder to let the right person find it with current music recommendation system where the most popular has more publicity. Therefore, the problem can be eliminated by building a music recommendation system that takes its genre and user's preference as the priority so that more exposure is given to different kind of songs. Based on the aforementioned problems, this paper proposes a music recommendation application that uses fuzzy inference engine to recommend songs to the user. The system should be able to correctly identify the user's preferences, timing, and certain qualities. Next, the system will process and recommend songs from its library to match the user's emotion and preference. It should also contain basic music playing functions as part of the requirements.

This paper proposes a music recommendation system based on fuzzy inference engine that considers user activities and emotion as part of the recommendation parameters. The system includes building a smart music recommendation system that has user profiling capabilities to recommend correct songs based on the user's preferences, mood and time. The remainder of this paper is organized as follows. Section 2 presents the research methodology to develop the music recommender system, Section 3 presents the evaluation results and analysis, and finally Section 4 concludes with some indication for future work.

\section{RESEARCH METHODOLOGY}

This paper proposes a music recommendation system based on fuzzy logics, called the Heartbeats, There are two major features within this system. The first feature is the audio-playing application. The application should be able to keep a list of record about current trending music and provide downloads to the tracks. Basic music-playing functionality is supported to offer the same kind of experience with current music playing applications. The second major feature of the system is the fuzzy inference system [15-17]. The fuzzy inference system is responsible to make a decision about music recommendations based on the user's music listening habits as well as expert knowledge about music genres and their effects on humans. The user's preference data is fed into the fuzzy inference system to obtain a decision that returns a score corresponding to the music track. The top ten music tracks with the highest recommendation score will be chosen as recommendations [18-20].

Figure 1 shows the system flow of Heartbeats. As shown in the figure, the main part of the system is made up of the fuzzy logic system. As the system receive the user's schedule and music listening preferences, the input data undergoes fuzzification, which is a process to convert input data into a normalised value for fuzzy logic. This process is done by setting membership functions for each input variable. For example, the 
condition whether the music track is fall within the preferred year range is either 0 or 1.The fuzzification of input data is followed by the inference engine, which is a logic interpreter based on fuzzy rulesets. The fuzzy ruleset is built upon the domain research in music theory, so it is equivalent to an advice of an expert. The ruleset will decide the final output based on the combination of input variables. It is achieved by checking the data of input variables and determine which fuzzy rule to fire. Therefore, the inference engine should accommodate each possible combination the input data can offer. Finally, the output data of the fuzzy inference system is then undergone defuzzification to obtain the music recommendation.

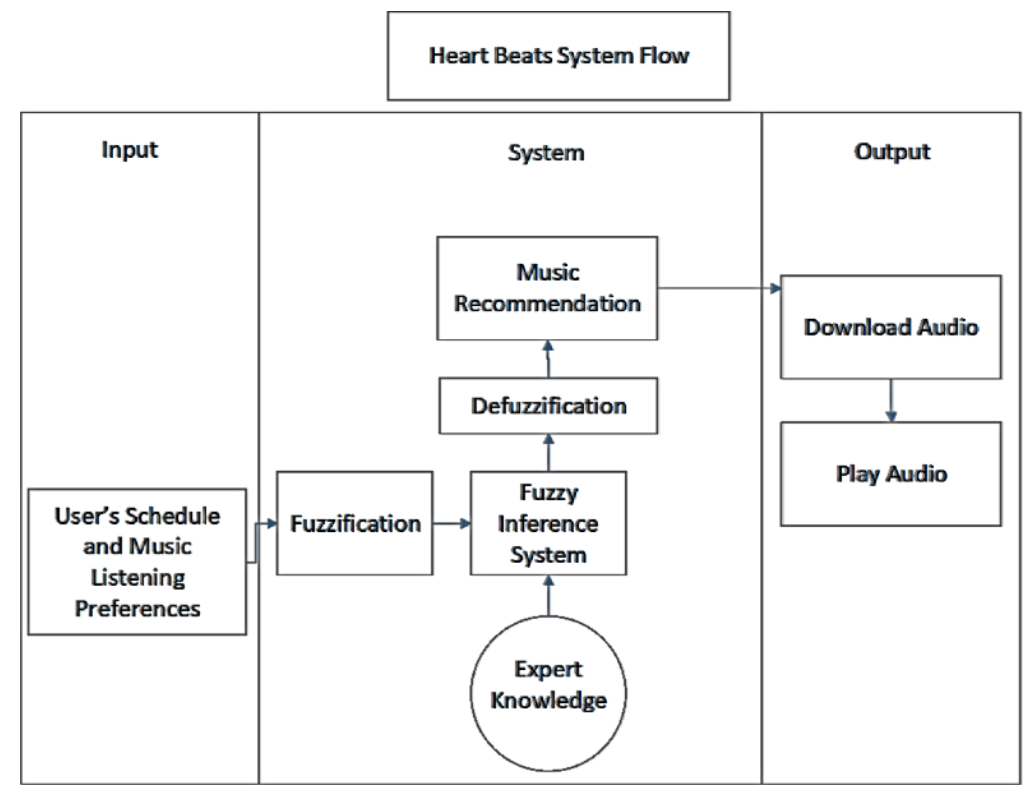

Figure 1. System flow of the music recommender system

Defuzzification is an inverse operation of the fuzzification process, where it transforms fuzzy sets into crisp sets which is quantifiable. The fuzzy inference system used for music recommendation in Heartbeats consists of five inputs variables, one output variable and 14 rules. All five input data are generated by the system with the information of user's preference on music listening habits and the music track.

\subsection{Input Variables}

There are five input variables in Heartbeats, which include the listening mode, preference, focus, relaxation mode, and year.

a. Listening Mode: Listening Mode is the preference option determined by the user's music listening habits. Its value changed depends on the purpose of the music listening.

b. Preference: Preference is the degree of the music track overlapping with the user's preferred genres. Depending on how many preferred genres is present in the music track the value is changed accordingly.

c. Focus: Focus is the degree of the music track overlapping with the genres that improve the listeners' focus. Depending on how many focus genres is present in the music track the value is changed accordingly.

d. Relax: Relax is the degree of the music track overlapping with the genres that helps the listeners to relax. Depending on how many relax genres is present in the music track the value is changed accordingly.

e. Year: Year is a condition whether the music track falls under the preferred year range set by the user. The result is obtained by comparing the created year of the music track and a predetermined year range set by the user.

\subsection{Output Variable}

Score is the only output variable in this fuzzy inference system. It indicates the degree of recommendation of the music track. The higher the value the more likely it is recommended to the user. It comes with five membership functions representing five different levels of likeliness. The output will be used by the system to dictate which music track is recommended to the user. Figure 2 shows the membership function for score. 


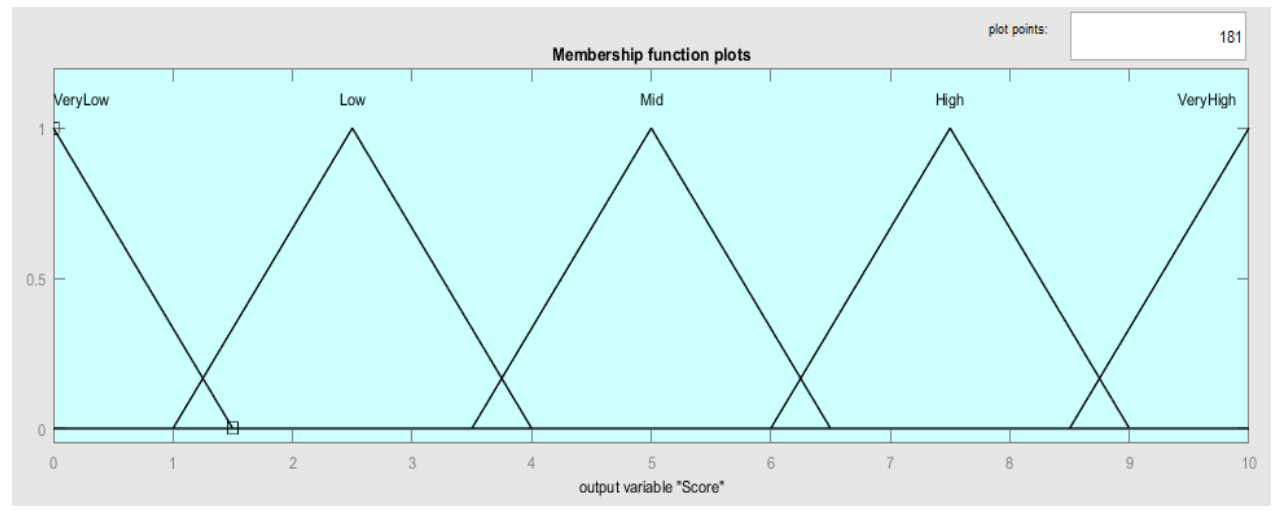

Figure 2. Membership function for output variable, which is score

\subsection{Rules}

There is a total of 14 rules created for the fuzzy inference system. The rules generally is created with one or two input variables and one output variable. The rules then collectively decide the final value of the output variable based on which rules are fired according to the input. The input variable ListeningMode is the variable that dictates the weight of the value of Focus, Relax and Preference. Figure 3 shows the each rule that involves Focus, Relax and Preference has ListeningMode as a secondary condition to determine whether the rule should be fired or not. This means each rule that involves Focus, Relax and Preference has ListeningMode as a secondary condition to determine whether the rule should be fired or not. Therefore, a music track that is suitable for focus tasks might not have a high recommendation score, if the ListeningMode is set to Relax.

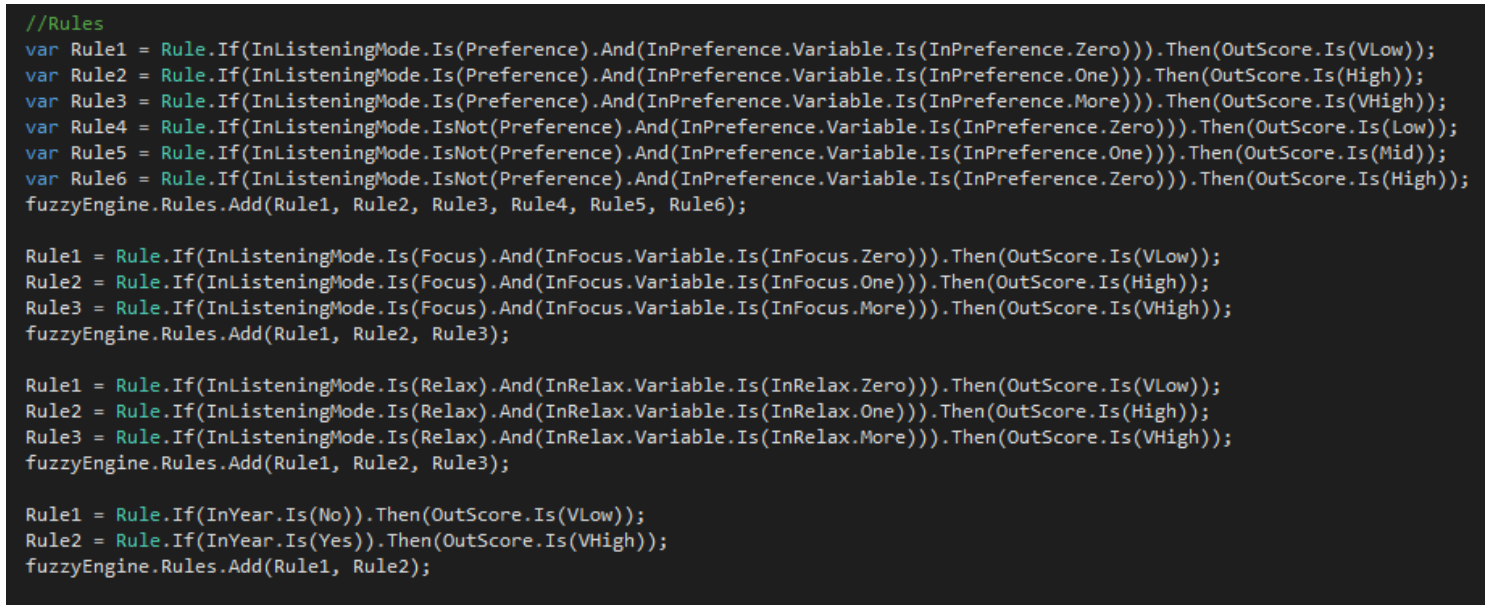

Figure 3. Code snippet to create rules using fuzzy inference system

A total of 12 rules is created for this fuzzy logic. The last input variable is Year, which only uses itself as the sole condition. It is a very straightforward rule that it increases the score if it falls within the year range, but decreases it if it does not. The last 2 rules are created for this variable, which forms 14 rules in total. Table 1 shows the sample output obtained from the fuzzy inference system.

Table 1. Sample output of the fuzzy inference system

\begin{tabular}{cccccr}
\hline Listening Mode & Preference & Focus & Relax & Year & Score \\
\hline Preference & One & One & Zero & Yes & 8.75 \\
Preference & More & Zero & One & Yes & 10.00 \\
Focus & One & Zero & One & No & 1.00 \\
Focus & One & More & One & No & 5.00 \\
Relax & More & One & One & Yes & 8.75 \\
\hline
\end{tabular}


From the sample output, it can be concluded that the second sample data is the most recommended music track based on the fuzzy logic, followed by the first and fifth sample data. The system operates based on the output information to recommend the best music track for the user after applying the domain logic in music psychology in the form of fuzzy logic.

\section{RESULTS AND ANALYSIS}

Heartbeats was developed with $\mathrm{C \#}$ as the programming language to develop the music recommendation system. To accommodate the language, Visual Studio is picked as the IDE of the software development. Libraries and tools are researched and picked for implementation of music-playing functionalities and data storage capability. The method of data storage for the music recommendation system is using Excel file because there is only one information to save, which is the users' personalized data. Lastly, Windows is chosen as the operating system for deployment of the final product.

\subsection{System Interface}

Figure 4 shows the interfaces of Heartbeats music recommender system. This page will display the recommended music once the user clicks on the "Load Audio" button. The user can then select a music track from the music list and click on "Play" to play the song. Other functions from audio playing application such as "Pause", "Rewind", "Stop" and volume control is available to adjust the audio feedback. The user is also able to navigate back to the previous page by clicking the "Back" button. The interface also allow users to choose between three presets of music listening preferences which will recommend music tracks to user based on a pre-set metric.
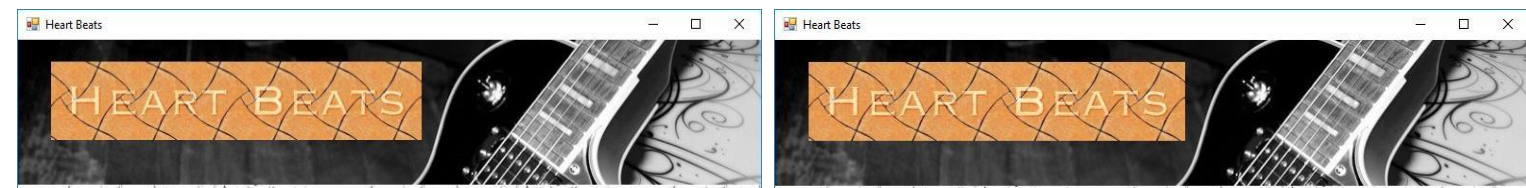

Pick a Prese

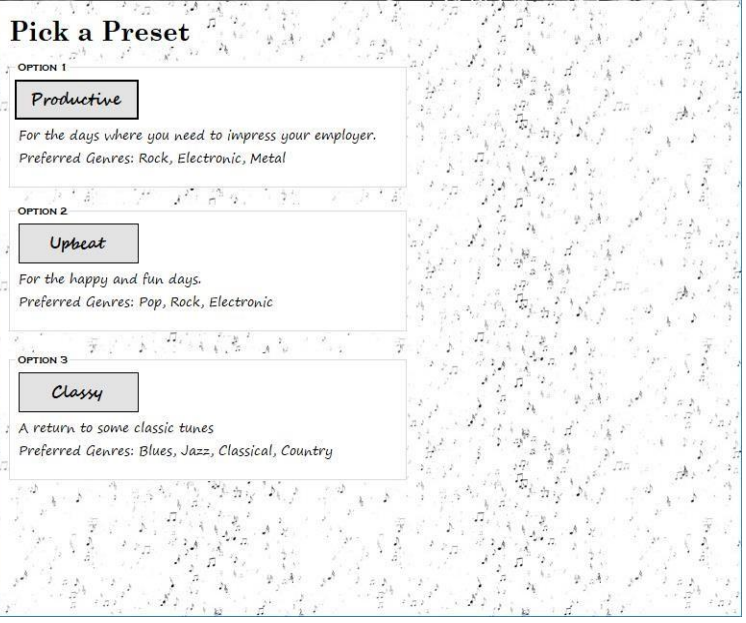

Figure 4. Interface of heartbeats

\subsection{User Acceptance Testing}

In order to evaluate the developed user profiling system, Heartbeats, a User Acceptance Testing (UAT) was carried out. UAT allow the developer to present the product to the user and obtained feedback and discover errors that normal testing procedures fail to catch. A total of 5 questions were posed to 30 users who participated in the UAT. The set of questions cover responses about user expectations, performance of audio quality produced by the system, system flow and interface design, and finally functionality issues. Figure 5 to Figure 9 show the UAT results and the findings.

According to Figure 5, 25 users find the system manage to achieve music recommendation that matches their preferences or current mood. This shows that the fuzzy logic managed to fulfil its objectives. Next, Figure 6 shows the feedback on the quality of music player. 
How is the design of the interface?

30 responses

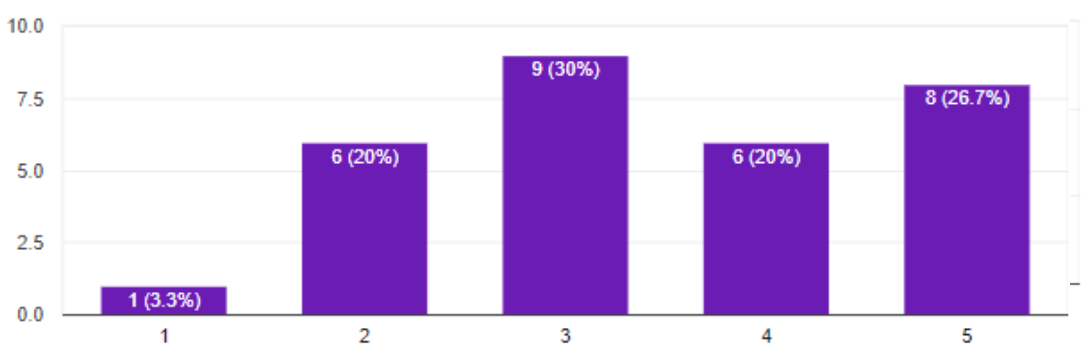

Figure 5. General user expectation

How is the audio quality of the music player?

30 responses

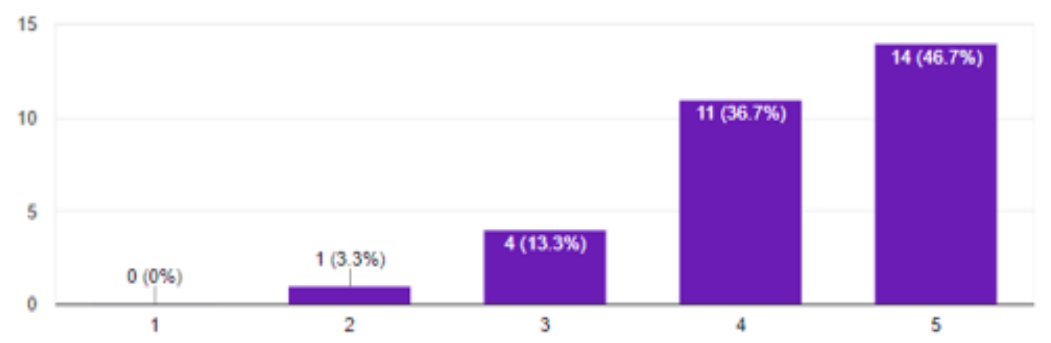

Figure 6. Performance of audio quality produced by the system

According to Figure 6, the music playing function managed to provide the best listening experience to the users with 25 respondents reply with positive feedback. Figure 7 shows the feedback on the system flow.

According to Figure 7, the opinion on the system flow is divided. Most of the user is neutral towards the workflow and 14 user likes the current system workflow. However, there is 6 users that shows dislike towards the current system workflow. The problem could be the stemming from the fact that the system does not load the recommendation immediately when transition towards the audio playing page and required a single button press to perform the loading task. Next, Figure 8 shows the opinions on the interface design.

According to Figure 8, the opinion on the system interface design is also divided. Many of the user thinks that the interface is clear and present information clearly in the main page. However, the audio playing page is missing certain information such as music track genre and current condition for music recommendation. Next, Figure 9 shows user feedback on problems faced when using the application.

How is the system flow?

30 responses

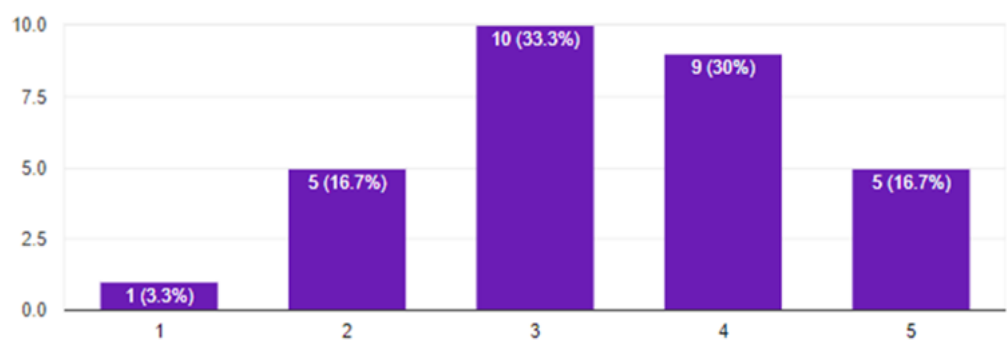

Figure 7. System flow 
How is the design of the interface?

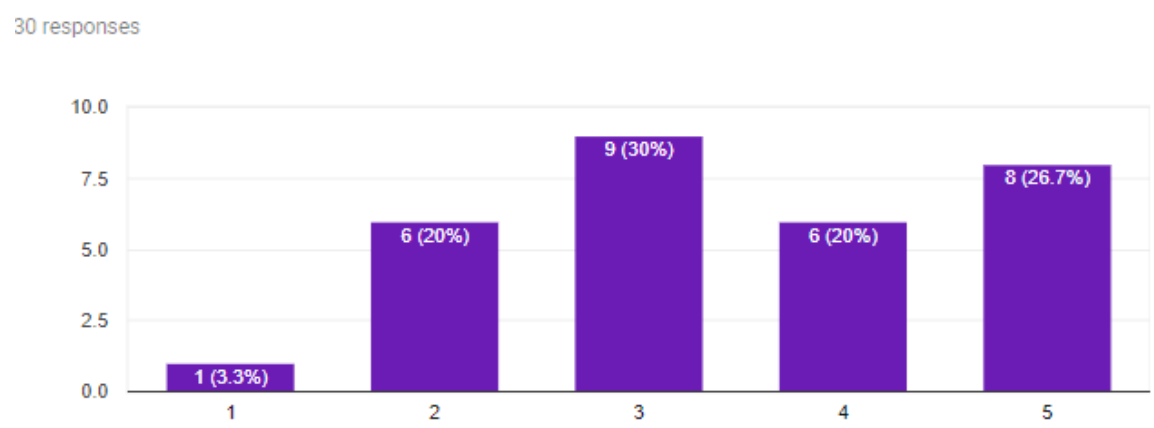

Figure 8. Design of the interface

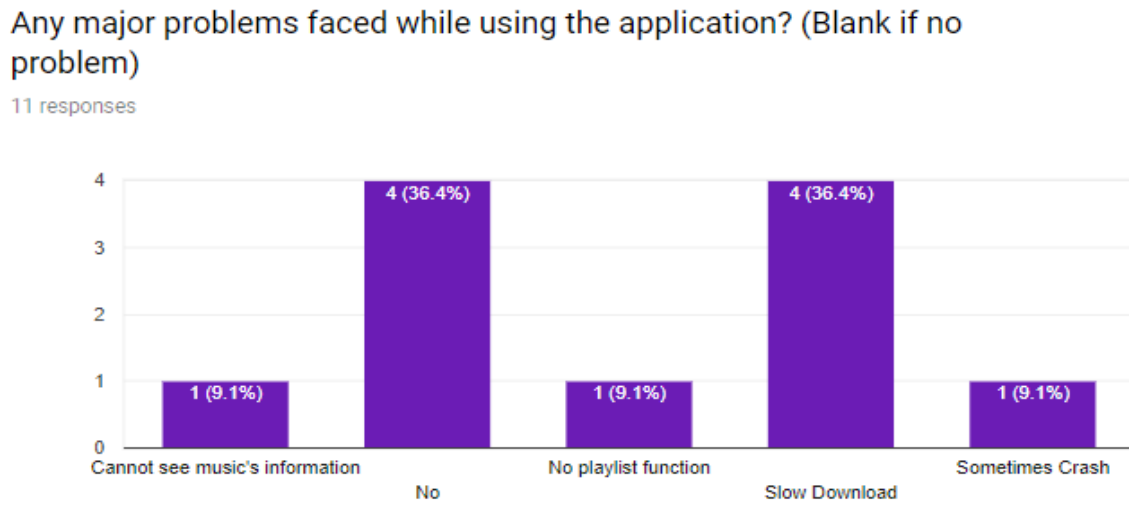

Figure 9. Functionalities problems encountered

The question in Figure 9 enforces all previous feedback towards the system. The system is shown has less audio playing functions such as playlist compare to current audio playing application. The download speed of the audio track is also slow and dependant on Wi-Fi connection. It is also reported to have unknown crashes which could not be solved during the UAT session.

One major benefit of Heartbeats is its scalability where new fuzzy rules can be incrementally added to the engine to further enhance the quality of its music recommendation. For example, more music tracks can be added to its library to enrich its music selection. Heartbeats also allow new lifestyle pre-sets to be created or customized in order to obtain different music recommendation [21-23]. There are three lifestyles options available for the user to choose, which can result in different music recommendation $[24,25]$. Users can benefit from this feature to personalize their music listening preferences. Heartbeats updates the music selection on daily basis, up to current trends and consist up to eight genres. The vast diversity of the music selection allows the fuzzy logic to apply its recommendation algorithm to more samples which can improve the accuracy of the result. Large selection of music also ensure that music recommendation is diverse and cater to a bigger audience. The audio playing application within Heart Beats has great audio quality and convenient functions. These features further enhance the music listening experience of the user.

\section{CONCLUSIONS}

This paper presented a prototype implementation of a music recommender system called the Heartbeats. Heartbeats interpret user's schedule and task to assess the user's music listening preferences. The system then used a fuzzy inference module that apply fuzzy logics in music recommendation to obtain result that can satisfy user music listening experience. The user experience is also improved by building an audio playing music application that allows the user to listen to the music tracks. Findings of the this paper have shown that Heartbeats's fuzzy inference engine has successfully achieved its aim, which is to improve users' music listening experience by giving suitable song recommendation based on user context situation. The system accomplishes this task by converting user's activity and mood into metrics that are used for music recommendation. 
In the future, more rules are to be added into the fuzzy inference system to increase the accuracy in music recommendation. Full user profile customization will then be implemented to allow user to freely alter the conditions and parameters affecting the algorithm. The audio playing music application can also support other quality of life features such as auto-play, playlist, song downloads and more. Finally, Heartbeat can be deployed in other platforms such as a web application or a mobile application.

\section{REFERENCES}

[1] Greenberg DM, Müllensiefen D, Lamb ME, Rentfrow PJ. "Personality predicts musical sophistication." Journal of Research in Personality. Oct 1;58:154-8, 2015.

[2] Swaminathan S, Schellenberg EG. "Current emotion research in music psychology." Emotion review. Apr;7(2):18997, 2015

[3] Karageorghis CI, Hutchinson JC, Jones L, Farmer HL, Ayhan MS, Wilson RC, Rance J, Hepworth CJ, Bailey SG. "Psychological, psychophysical, and ergogenic effects of music in swimming." Psychology of Sport and Exercise. Jul 1;14(4):560-8, 2013.

[4] Palit HC, Aysia DA. The effect of pop musical tempo during post treadmill exercise recovery time." Procedia Manufacturing. Vol 1, no 4, pp. 17-22, 2015.

[5] Wu PY, Huang ML, Lee WP, Wang C, Shih WM. "Effects of music listening on anxiety and physiological responses in patients undergoing awake craniotomy." Complementary therapies in medicine. Jun 1;32:56-60, 2017.

[6] Sen A, Larson M. "From Sensors to Songs: A Learning-Free Novel Music Recommendation System using Contextual Sensor Data.” In LocalRec@RecSys, Sep 19, pp. 40-43, 2015.

[7] Boothby DM, Robbins SJ. "The effects of music listening and art production on negative mood: A randomized, controlled trial". The Arts in Psychotherapy. Jul 1;38(3):204-8, 2011.

[8] Houston Behavioral, "The Top 3 Causes of Stress in Today's World" http://www.houstonbehavioralhealth.com/blog/top-3-causes-of-stress (accessed April 11, 2019).

[9] Wu PY, Huang ML, Lee WP, Wang C, Shih WM. Effects of music listening on anxiety and physiological responses in patients undergoing awake craniotomy. Complementary therapies in medicine. Jun 1;32:56-60, 2017.

[10] Liu YH, Lee CS, Yu CH, Chen CH. " Effects of music listening on stress, anxiety, and sleep quality for sleepdisturbed pregnant women.” Women \& health. Apr 2;56(3), 296-311, 2016.

[11] Raju MK, Shafiulilah SK. "An Enhanced Risk Prediction System for Cardiovascular Disease in India using Fuzzy Classification”. International Journal of Computer Applications. Jan 1;975:8887, 2015.

[12] Majma N, Babamir SM, Monadjemi A. "Runtime verification of pacemaker functionality using hierarchical fuzzy colored Petri-Nets". Journal of medical systems. Feb 1;41(2), pp. 27, 2017.

[13] Natividad MC, Gerardo BD, Medina RP. "A fuzzy-based career recommender system for senior high school students in K to 12 education”. “, Feb Vol. 482(1), pp. 012025, 2019.

[14] Ali F, Islam SR, Kwak D, Khan P, Ullah N, Yoo SJ, Kwak KS. "Type-2 fuzzy ontology-aided recommendation systems for IoT-based healthcare". Computer Communications. 2018 Apr 1;119:138-55.

[15] Mohammed HA, Hagras H. Towards Developing "Type 2 Fuzzy Logic Diet Recommendation System for Diabetes". In2018 10th Computer Science and Electronic Engineering (CEEC) Sep 19, pp. 56-59, 2018.

[16] Nathan G, Mastronardi T, inventors; “"'TouchTunes Music Corp, assignee. Jukebox entertainment system having multiple choice games relating to music". United States patent US 9,919,214, Mar 20, 2018.

[17] O'Neill DM, Boone K, Del Beccaro DJ, inventors "Music Choice, assignee. System for providing music content to a user." United States patent application US 10/219,027, Feb 26, 2019.

[18] Braasch J, Deshpande N, Oliveros P, Bringsjord S, inventors; Rensselaer Polytechnic Institute, assignee. "Interactive, expressive music accompaniment system". United States patent application US 10/032,443, Jul 24, 2018.

[19] McClard AP, Knier A, inventors; Intel Corp, assignee. "Music teaching system". United States patent application US 10/078,969, Sep 18, 2018.

[20] Katarya R, Verma OP. "Efficient music recommender system using context graph and particle swarm". Multimedia Tools and Applications,Jan 1, 77(2), pp. 2673-87, 2018.

[21] Milne JR, Carlsson GP, Richman SM, Zustak FJ, inventors; Sony Corp, assignee. "Audio speaker system with virtual music performance". United States patent US 9,866,986. Jan 9, 2018.

[22] Debique K, inventor; Sync Floor Inc, assignee."System and Method for Natural Language Music Search". United States patent application US 15/881,416, Jan 10, 2019.

[23] Bussigel P, Rovan J, inventors; Brown University, assignee."System for electronically generating music". United States patent application US 15/996,406, Sep 27, 2018.

[24] Covarrubias M, Aruanno B, Cianferoni T, Rossini M, Komarova S, Molteni F. "Neuro Rehabilitation System Through Virtual Reality, Music and Fragrance Therapy". InInternational Conference on NeuroRehabilitation 2018 Oct 16, pp. 848-852.

[25] Hsieh K, Lin B, Yuan Y, Yu S, inventors; 1More Inc, assignee. "Method and system for providing a social service based on music information". United States patent application US 10/078,692,. Sep 18, 2018. 